\title{
ON AZIMUTHAL VARIATION OF DISPLACEMENTS OF NORMAL MODE SOLUTIONS OF SH TYPE EXCITED BY A DOUBLE-COUPLE POINT SOURCE WITH SPECIAL ATTENTION TO THE MODE-RAY RELATION
}

\author{
Toshikazu ODAKA \\ Earthquake Research Institute, the University of Tokyo, Tokyo
}

(Received January 7, 1972)

The normal mode solution for Love waves from a shear fault in a stratified layer over the half-space is investigated with special attention to the azimuthal behavior of amplitude compared with the radiation pattern of $S H$ waves. It is found that Love waves are denoted by the sum of two terms associated with a particular set of rays of physical significance. For the torsional oscillation of a homogeneous elastic sphere due to the shear fault, a similar relation is recognized between higher radial modes and rays. The mode-ray relations obtained in the above two cases coincide with the ones derived in other literature from quite different considerations that interpret the normal modes by interference phenomena.

The radiation patterns of $S H$ waves and of normal modes are found to be related through the apparent equivalent take-off angle $\tau_{h}$, which is defined so that the radiation pattern of square amplitude for a normal mode may be equal to the pattern of the sum of the square amplitudes for SH waves emitted in the two directions specified by the take-off angles $\bar{\tau}_{h}$ and $\pi-\tau_{h}$. Provided that the apparent equivalent take-off angles are known beforehand as a function of the eigen-frequency and the focal depth, it is easy to obtain the azimuthal dependence of amplitude of the normal modes excited by a shear fault of arbitrary dip and slip, because the azimuthal behavior is the same as the radiation pattern of $S H$ waves associated with the apparent equivalent take-off angles. Numerical experiments are shown for the fundamental torsional oscillation of a Gutenberg-Bullen $A^{\prime}$ spherical earth excited by a pure dip slip and a pure strike slip fault at depth $5.35 \mathrm{~km}$.

\section{Introduction}

Attempts at revealing the mode-ray relation have been made by many authors. BRUNE (1964) proposed an excellent idea of connecting rays and normal modes of a spherical earth and applied the method successfully for obtaining torsional higher mode dispersion curves from body wave phases. BEN-MENAHEM (1964) also revealed the mode-ray duality from the analytical considerations. The contribution of theoretical seismograms to the study of this problem is significant and valuable results have 
been achieved (e.g., Alterman and Kornfeld, 1966; Alterman and Aboudi, 1969; LANDISMAN et al., 1970).

It is well known that the radiation pattern of the amplitude of normal modes, either of surface waves propagating on the surface of multi-layered elastic half-space or of free vibrations of an elastic sphere, depends on the focal mechanism and the focal depth. It is thus reasonable to suppose that the radiation pattern for normal modes has some correlation with that for body waves radiated from the same source if some mode-ray correspondence relation exists.

In this paper, it is examined whether we can find some evidence revealing the mode-ray relation in the expression of normal mode solutions of $S H$ type due to a double-couple force system. The method is based on the idea that if there is a certain relationship between modes and rays, the azimuthal variations of amplitude for a normal mode and for body waves associated with a particular ray must show identical patterns. The procedure is realized by investigating expressions for normal modes and for body waves due to a source with some directivity, referring to their radiation patterns.

Two simple cases are studied. One is Love waves propagating in a layer over a semi-infinite medium and the other the torsional oscillation of a homogeneous elastic sphere. Certain correspondence relations between normal modes and rays are found; these are consistent with those pointed out in other literature discussing similar problems. Useful formulas for obtaining the radiation pattern of amplitude spectra of normal mode solutions are also derived.

\section{Expressing Radiation Pattern of $S H$ Waves}

Displacements of body waves due to a shear fault in the infinite homogeneous isotropic elastic medium have been formulated by many investigators (e.g., BeNMeNAHEM et al., 1965; SATo, 1969). These are required in later sections for comparing with radiation patterns of normal modes.

For horizontally polarized transverse waves due to a fault of arbitrary dip and slip, adopting only the far-field term, we have

$$
u_{S H}\left(R, \varphi, i_{h}, t\right)=\frac{M_{0}}{4 \pi \rho v_{S}^{8}} \cdot R_{S H} \cdot \dot{f}\left(t-R / v_{S}\right) / R
$$

where $R$ denotes the distance between the source and the station, $i_{h}$ the take-off angle at the focus, $\varphi$ the azimuth of the station measured from the strike direction, $t$ the time, $M_{0}$ the moment of the force system equivalent to the shear fault, $\rho$ the density, and $v_{S}$ the shear wave velocity. The source time function is $f(t)$, and a dot $(\cdot)$ over it means $d / d t$.

$R_{S H}$ stands for a factor indicating the radiation pattern of $S H$ waves and is given as

$$
R_{S H}\left(i_{h}, \varphi\right)=-\left(d_{1} \cdot \cos \varphi-d_{2} \cdot \sin \varphi\right) \cos i_{h}-\left(d_{3} \cdot \cos 2 \varphi-d_{4} \cdot \sin 2 \varphi\right) \sin i_{h},
$$

where

$$
\left.\begin{array}{ll}
d_{1}=-\cos 2 \delta \cdot \sin \lambda, & d_{2}=-\cos \delta \cdot \cos \lambda, \\
d_{3}=\sin \delta \cdot \cos \lambda, & d_{4}=-(\sin 2 \delta \cdot \sin \lambda) / 2 .
\end{array}\right\}
$$


$\delta$ gives the dip angle and $\lambda$ the slip angle. The geometry of the fault is interpreted in Fig. 1.

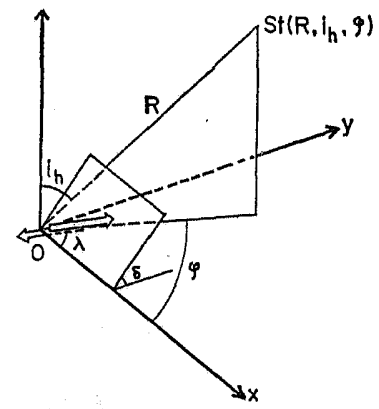

Fig. 1. Coordinate systems and source geometry. $O$ refers to the focus and $S t$ the station. Arrows indicate the direction of the fault movement along one of two possible infinitesimal fault planes associated with the double-couple force system located at the coordinate origin.

Employing the formulas

$$
\left.\begin{array}{l}
C_{1}=d_{1} \cdot \cos \varphi-d_{2} \cdot \sin \varphi \\
C_{2}=d_{8} \cdot \cos 2 \varphi-d_{4} \cdot \sin 2 \varphi
\end{array}\right\}
$$

the radiation pattern of $S H$ waves of Eq. (2) is reduced to

$$
R_{S H}\left(i_{h}, \varphi\right)=-C_{1} \cdot \cos i_{h}-C_{2} \cdot \sin i_{h} .
$$

This relation plays an important role in correlating modes and rays in later sections. The following formula is also useful for calculating the radiation pattern of normal modes of rotational type.

$$
\begin{aligned}
E_{S H}\left(i_{h}, \varphi\right) & =\left[R_{S H}^{2}\left(i_{h}, \varphi\right)+R_{S H}^{2}\left(\pi-i_{h}, \varphi\right)\right] / 2, \\
& =C_{1}^{2} \cdot \cos ^{2} i_{h}+C_{2}^{2} \cdot \sin ^{2} i_{h} .
\end{aligned}
$$

This quantity is concerned with the energy released from the source along the two rays having the same ray parameter.

\section{Love Waves and Rays}

In this section, Love waves excited by a shear fault are investigated assuming the simplest model, a single-layer and half-space model, with special reference to the azimuthal behavior of amplitude in comparison with that of $S H$ waves. This model is preferred because the analytical expression for solutions can be obtained with comparative ease and because the discussion analogous to the problem of sound wave transmission in shallow water, in which the mode-ray correspondence relation is well established from a viewpoint of interference phenomena, is possible as well.

HASKeLL (1964) treated the problem for the excitation of surface waves due to point sources in a multilayered medium with the aid of layered matrices. The solution relevant to the present problem is also involved in his study.

The azimuthal displacement on the surface far from the epicenter due to a shear fault within a layer is

$$
u_{\varphi}(r, \varphi, z=0)=-\frac{\kappa \cdot M_{0}}{2 \rho v s^{2} \cdot\left[\frac{\partial F(\xi)}{\partial \xi}\right]_{\xi=\kappa}}\left[\left(d_{1} \cdot \cos \varphi-d_{2} \cdot \sin \varphi\right)\left(e^{j \bar{\beta} d}-e^{-j \bar{\beta} d}\right)\right.
$$




$$
\begin{aligned}
& \left.+\frac{\kappa}{\bar{\beta}}\left(d_{3} \cdot \cos 2 \varphi-d_{4} \cdot \sin 2 \varphi\right)\left(e^{j \bar{\beta} d}+e^{-j \bar{\beta} d}\right)\right] \\
& \times \sqrt{\frac{2}{\pi \kappa r}} \cdot e^{-j(\kappa r-p t+\pi / 4)}
\end{aligned}
$$

where

$$
F(\xi)=1-\frac{\beta-\mu^{\prime} \beta^{\prime} \mid \mu}{\beta+\mu^{\prime} \beta^{\prime} / \mu} \cdot e^{-2 \beta H}
$$

and

$$
\beta=\sqrt{\xi^{2}-k^{2}}, \quad \beta^{\prime}=\sqrt{\xi^{2}-k^{\prime 2}}, \quad \bar{\beta}=\sqrt{k^{2}-\kappa^{2}}, \quad k=p / v_{S}, \quad k^{\prime}=p / v_{S^{\prime}},
$$

$M_{0}$ means the moment of a double-couple force system equivalent to the shear fault and $\rho, \mu$ and $v$ s the density, rigidity and shear wave velocity in the layer, respectively. The corresponding quantities in the semi-infinite medium are $\rho^{\prime}, \mu^{\prime}$ and $v s^{\prime} . H$ is the thickness of the layer, $d$ the depth down to the focus, $p$ the angular frequency, and $j$ the unit of imaginary numbers. The cylindrical coordinate system referred to the origin at the free surface is $(r, \varphi, z)$ as indicated in Fig. 2. The focal mechanism re-

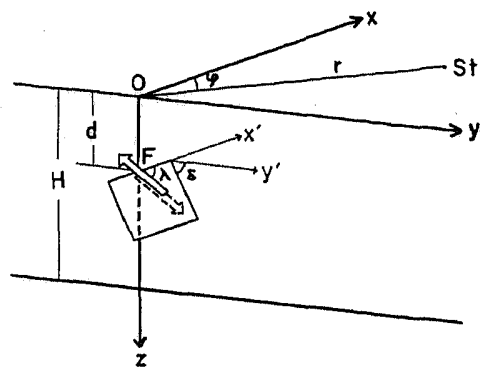

Fig. 2. A single-layer semi-infinite model. $F$ indicates the seismic origin. The $x-y$ plane constitutes the free surface.

ferring to the $(x, y, z)$ coordinate system shown in Fig. 2 is identical to the one in Fig. 1 except for the vertical position of the hypocenter.

$$
F(\xi)=0,
$$

gives the characteristic equation of Love waves, and $\kappa$ stands for the roots of the equation. The phase velocity of Love waves is denoted by

$$
C_{L}=p / \kappa,
$$

and is within the range

$$
v_{S} \leq C_{L} \leq v S^{\prime} .
$$

Equation (7) was obtained analytically from the source functions for dipolar sources given by SATo (1969) and therefore it seems to show a somewhat different expression from Haskell's. However, a slight modification of it immediately proves that both are identical.

Other components than the azimuthal displacement are disregarded because the 
vertical component is missing essentially and the radial one is negligibly small ${ }_{\mathbf{A}}$ compared to the azimuthal one at a far station.

Substituting Eq. (4) into Eq. (7),

$$
\begin{aligned}
u_{\varphi}(r, \varphi, 0) & =K \cdot\left[\left(-C_{1}-\frac{\kappa}{\bar{\beta}} \cdot C_{2}\right) \cdot e^{j \bar{\beta} d}+\left(C_{1}-\frac{\kappa}{\bar{\beta}} \cdot C_{2}\right) \cdot e^{-j \bar{\beta} d}\right] \cdot e^{j(p t-\kappa r)}, \\
& =2 K \cdot\left[\left(\frac{\kappa}{\bar{\beta}} \cdot C_{2} \cdot \cos \bar{\beta} d\right)^{2}+\left(C_{1} \cdot \sin \bar{\beta} d\right)^{2}\right]^{1 / 2} \cdot e^{j\left(p t-\kappa r+\phi_{L}+\pi\right)}
\end{aligned}
$$

where $K$ and $\phi_{L}$ are defined as

$$
\left.\begin{array}{l}
K=\frac{\kappa \cdot M_{0}}{2 \rho v \mathrm{~s}^{2} \cdot\left[\frac{\partial F(\xi)}{\partial \xi}\right]_{\xi=\kappa}} \cdot \sqrt{\frac{2}{\pi \kappa r}} e^{-j \pi / 4}, \\
\phi_{L}=\tan ^{-1}\left[\frac{C_{1}}{\frac{\kappa}{\bar{\beta}} \cdot C_{2}} \cdot \tan \bar{\beta} d\right] .
\end{array}\right\}
$$

It is interesting to examine the radiation pattern of each term in the brackets of Eq. (13), for these terms can be expected to correspond to two rays, one directed downward and one upward from the focus, respectively. It will then be obvious, referring to Eq. (5), that the azimuthal dependence of these terms is the same as of SH waves emitted in the directions with the take-off angles defined by

$$
\tan i_{h}^{x}= \pm \kappa / \bar{\beta} \text {. }
$$

The plus sign corresponds to the ray radiated downward from the source and minus the upgoing one, which are denoted in Fig. 3 as $R 1$ and $R 2$ respectively. Provided that the value of $i_{h}{ }^{x}$ is limited between 0 and $\pi / 2$, the solutions of Eq. (16) are given by $i_{h}{ }^{L}$ and $\pi-i_{h}{ }^{L}$. The angle $i_{h}{ }^{L}$ will be called the equivalent take-off angle for Love waves.

From Eqs. (9), (11), and (16), the following familiar relation is derived

$$
C_{L}=v_{S} / \sin i_{h}^{L} .
$$

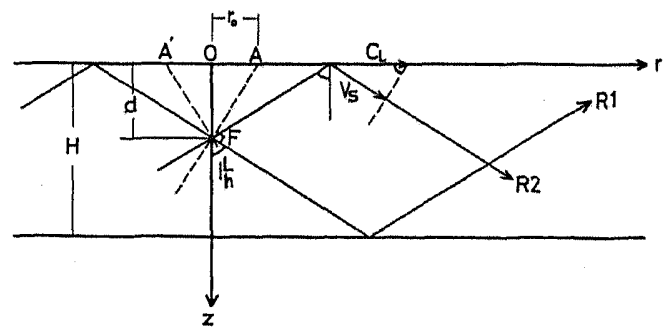

Fig. 3. Ray geometry interpreting the Love wave-SH wave correspondence relation. $R 1$ and $R 2$ indicate the two rays with common ray parameter. $C_{L}$ means the phase velocity of Love waves, $v_{\mathrm{s}}$ the shear wave velocity, and $i_{h}{ }^{L}$ the equivalent take-off angle for Love waves. 
This relation implies that the wave-front of plane $S H$ waves with incident angle $i_{h}{ }^{L}$ progresses along the surface with the phase velocity $C_{L}$ (cf. Fig. 3). The broken lines indicated by $A F$ and $A^{\prime} F$ show the wave-fronts associated with the rays $R 1$ and $R 2$.

It is evident from the figure that the distance $r_{0}$ between $O$ and $A$ or $A^{\prime}$ satisfies the relation

$$
\tan i_{h}{ }^{L}=d / r_{0},
$$

Employing Eqs. (5), (9), (11), (16) and (18), Eq. (13) can be reduced to

$$
\begin{aligned}
u_{\varphi}(r, \varphi, 0)= & \left(K / \cos i_{h}^{L}\right) \cdot\left[R_{S H}\left(i_{h}^{L}, \varphi\right) \cdot e^{-j \kappa\left(r-r_{0}-\sigma_{L} \cdot t\right)}\right. \\
& \left.+R_{S H}\left(\pi-i_{h}{ }^{L}, \varphi\right) \cdot e^{-j \kappa\left(r+r_{0}-C_{L} \cdot t\right)}\right] .
\end{aligned}
$$

The derivation is straightforward. This final expression, Eq. (19) of the normal mode solution is interesting from the viewpoint of connecting modes with rays. It reveals that the solution for Love waves consists of the sum of two waves with different azimuthal variations and initial phases. The former possesses the radiation pattern equivalent to that of $S H$ waves radiated in the direction of the take-off angle $i_{h}{ }^{L}$ and the initial phase $\kappa r_{0}$. For the latter, these quantities have values $\pi-i_{h} L$ and $-\kappa r_{0}$. Thus, the two features with regard to the radiation pattern and the initial phase support that the two waves correspond, respectively, to the downgoing and upgoing rays at the focus with the same ray parameter specified by the equivalent take-off angle $i_{h}{ }^{L}$.

It should be pointed out that the situation stated above is not exactly the one that actually occurs near the source region, because the discussion in this section holds under the condition that the observing point is enough apart from the epicenter that the plane wave approximation is valid for the spherical waves.

The particular set of rays assigned by Eq. (16) or (17) is very important from the viewpoint of the physical interpretation of normal modes by the ray theory, because the characteristic equation denoted by Eq. (10), which permits calculation of the dispersion curves of Love waves for the layered model in question, can also be derived by considering the constructive interference effects of downgoing and upgoing waves associated with this particular set of rays. For the sound wave transmission in the layered liquid space, these aspects are illustrated in detail in other literature (e.g., EwING et al., 1957; OFFICER, 1958) and for Love waves by SATÔ (e.g., 1951, 1954).

Thus, it is found that the comparison of the radiation patterns of body waves and of normal modes brings out satisfactory results for connecting the particular rays of physical significance with a normal mode.

Next the dependence of Love wave displacements on the azimuthal angle is considered; it differs from either of the component waves, i.e., the downgoing or upgoing waves, as is apparent from Eq. (19). Equation (14) reveals that the radiation pattern of amplitude spectra of Love waves depends on the focal depth as well as on the period. Its pattern has some connection with that of $S H$ waves, which can be derived from Eqs. (6) and (14). If the take-off angle $\bar{\tau}_{h}{ }^{L}$ is defined as

$$
\tan ^{2} \bar{\iota}_{h}{ }^{L}=(\kappa / \bar{\beta})^{2} \cdot \cot ^{2} \bar{\beta} d,
$$

it is obvious that the Love wave radiation pattern is given by

$$
R_{L}(d, \varphi)=\left[E_{S H}\left(\bar{\tau}_{h}^{L}, \varphi\right)\right]^{1 / 2} \cdot\left|\sin \bar{\beta} d / \cos \bar{\tau}_{h}{ }^{L}\right| .
$$

The absolute value of Eq. (14) is then reduced to 


$$
\left|u_{\varphi}(r, \varphi, 0)\right|=2 \cdot|K| \cdot R_{L}(d, \varphi) .
$$

The apparent equivalent take-off angle for Love waves, $\tau_{h}{ }^{L}$, provides some advantages in calculating the radiation pattern of Love waves since it can be realized by evaluating only that of $S H$ waves. From Eqs. (21) and (22), it is found that the pattern for the square amplitude of Love waves shows similar feature to that for $S H$ waves given by Eq. (6).

The two angles $i_{h}{ }^{L}$ and $\bar{l}_{h}{ }^{L}$ differ essentially because the former is inherent in respective normal modes while the latter varies as a function of the focal depth even for any assigned modes.

\section{Toroidal Modes and Rays}

The method developed in the preceding section revealed the mode-ray correspondence relation for the single-layer and half-space model. In this section, the method is applied to the torsional oscillation of a homogeneous isotropic elastic sphere. It is, then, expected that a mode-ray relation consistent with the one pointed out by BENMenahem (1964) will be derived.

Analytical expressions for the torsional displacement from the shear fault of arbitrary dip and slip in the homogeneous sphere are obtained without difficulty with the aid of equivalent source functions of the double-couple force system introduced by UsAMI et al. (1970). The equivalent source functions are defined as apparent discontinuities of displacements and stresses on the concentric spherical surface, on which the source is located, caused by the original source functions.

The equivalent source functions of the torsional modes for the shear fault indicated in Fig. 4 are

$$
\begin{aligned}
& \Delta u=0, \\
& \Delta v=-\frac{M_{0}}{4 \pi \mu b^{2}} \cdot\left(d_{1} \cdot \sin \varphi+d_{2} \cdot \cos \varphi\right) \cdot e^{j p t} \cdot \sum_{n} \frac{2 n+1}{n(n+1)} \cdot \frac{P_{n}{ }^{1}(\cos \theta)}{\sin \theta}, \\
& \Delta w=-\frac{M_{0}}{4 \pi \mu b^{2}} \cdot\left(d_{1} \cdot \cos \varphi-d_{2} \cdot \sin \varphi\right) \cdot e^{j p t} \cdot \sum_{n} \frac{2 n+1}{n(n+1)} \cdot \frac{d P_{n}{ }^{2}(\cos \theta)}{d \theta}, \\
& \widehat{\Delta r r}=0, \\
& \widehat{\Delta r \theta}=\frac{M_{0}}{4 \pi b^{8}} \cdot\left(d_{3} \cdot \sin 2 \varphi+d_{4} \cdot \cos 2 \varphi\right) \cdot e^{j p t} \cdot \sum_{n} \frac{2 n+1}{n(n+1)} \frac{2 P_{n}^{2}(\cos \theta)}{\sin \theta} \\
& \widehat{\Delta r \varphi}=\frac{M_{0}}{4 \pi b^{8}} \cdot\left(d_{3} \cdot \cos 2 \varphi-d_{4} \cdot \sin 2 \varphi\right) \cdot e^{j p t} \cdot \sum_{n} \frac{2 n+1}{n(n+1)} \frac{d P_{n}^{2}(\cos \theta)}{d \theta}
\end{aligned}
$$

where $M_{0}$ means the moment of the double-couple force system equivalent to the shear fault; $b$ the radius of the spherical surface where the source is located; $\mu$ the rigidity; $(r, \theta, \varphi)$ the polar coordinates referring to the center of the sphere; $u, v$, and $w$ the radial, colatitudinal, and azimuthal displacements; $\overparen{r r}, \widehat{r \theta}$, and $\widehat{r \varphi}$ the radial, colatitudinal, and azimuthal stress components; and $P_{n}{ }^{m}(\cos \theta)$ the associated Legendre function. Equation (3) defines $d_{1}, d_{2}, d_{3}$, and $d_{4}$, and they depend on dip and slip angles of the fault.

Some caution is taken with the derivation of Eq. (23) since the applied double 


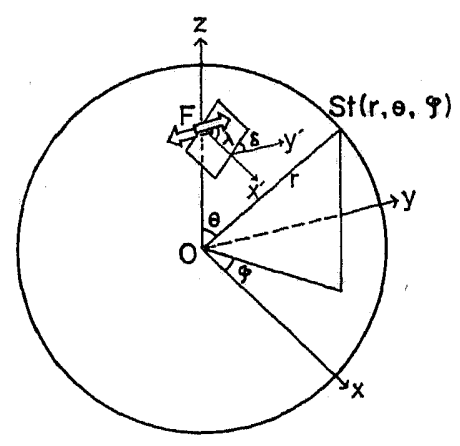

Fig. 4. Source geometry and the spherical coordinate system, $O$ is the center of the sphere, $F$ the focus, and $S t$ the station.

couple forces are different in their directions from those assumed in the paper of UsAmI et al. (1970). The solution was obtained according to the second method of the two expressed in the paper by UsAmr et al. (1970, p. 547). This requires the supplementary boundary condition to have discontinuities of displacement and stress on the source surface amounting to the values given by the equivalent source functions in Eq. (23) in addition to the conditions at the free surface and at the center of the earth. The expression for the azimuthal displacement at the free surface is

$$
\begin{aligned}
w(a, \theta, \varphi, t)= & -\frac{j M_{0}}{2 \pi \mu a^{2} b} \cdot \sum_{i, n} \frac{2 n+1}{n(n+1)}\left\{\frac { f ^ { * } ( p ) \cdot e ^ { j p t } } { \frac { \partial G _ { n } ( a ) } { \partial p } } \left[C_{1} \cdot b G_{n}(b) \cdot \frac{d P_{n}{ }^{1}(\cos \theta)}{d \theta}\right.\right. \\
& \left.\left.+C_{2} \cdot j_{n}(k b) \cdot \frac{d P_{n}^{2}(\cos \theta)}{d \theta}\right]\right\}_{p=i_{i} p_{n}},
\end{aligned}
$$

where

$$
G_{n}(r)=\frac{d j_{n}(k r)}{d r}-\frac{j_{n}(k r)}{r}=r \frac{d}{d r}\left(\frac{j_{n}(k r)}{r}\right) .
$$

$f^{*}(p)$ means the Fourier transform of the source time function, $j_{n}(\xi)$ the spherical Bessel function of order $n, i p_{n}$ the angular eigen-frequency associated with the radial mode number $i$ and the colatitudinal order number $n, k$ the wave number, and $a$ the radius of the earth. $C_{1}$ and $C_{2}$ are defined by Eq. (4).

$G_{n}(a)=0$ is the characteristic equation for the torsional oscillation of the homogeneous sphere, and the solution of the equation gives discrete eigen-frequencies as a function of mode and order numbers. The numerical solution is, for example, given by SATÔ and UsAMI (1962) up to considerably high modes.

There is no radial displacement for the toroidal modes. The colatitudinal component is disregarded because it shows much smaller amplitude than the azimuthal.

In the following, only the quantity in brackets in Eq. (24) is considered, for it is all that governs the azimuthal variation of the displacement spectra for the torsional oscillation. In the following, $i p_{n}$ will be simply written as $p$.

Employing the asymptotic formulas for the associated Legendre function, this quantity is, for order numbers $n \gg 1$, reduced as follows

$$
C_{1} \cdot b G_{n}(b) \cdot \frac{d P_{n}{ }^{1}(\cos \theta)}{d \theta}+C_{2} \cdot j_{n}(k b) \cdot \frac{d P_{n}{ }^{2}(\cos \theta)}{d \theta}
$$




$$
\begin{aligned}
& \approx n^{2} \cdot k_{n} \cdot\left[C_{1} \cdot b G_{n}(b) \cdot \sin \Theta-C_{2} \cdot n j_{n}(k b) \cdot \cos \Theta\right] \\
& =\left(n^{2} \cdot k_{n} / 2\right)\left[\left\{j \cdot C_{1} \cdot b G_{n}(b)-C_{2} \cdot n j_{n}(k b)\right\} e^{-j \Theta}+\left\{j \cdot C_{1} \cdot b G_{n}(b)+C_{2} \cdot n j_{n}(k b)\right\} e^{j(\Theta+\pi)}\right],
\end{aligned}
$$

where

$$
\Theta=(n+1 / 2) \theta+\pi / 4, \quad k_{n}=\sqrt{2 / n \pi \sin \theta}, \quad(0<\theta<\pi) .
$$

In the last expression of Eq. (26), the first term in the brackets corresponds to waves propagating in the $+\theta$ direction and the second in the $-\theta$. These aspects were proved by the numerical calculations for the fundamental spheroidal modes of a radially heterogeneous sphere (ODAKA and USAMI, 1970). For any toroidal modes as well, we assume that the summation of the contribution from the first term gives rise to waves propagating in the $+\theta$ direction and the second term in the opposite direction. Hereafter, attention will be paid only to the first term, that is, waves progressing in the $+\theta$ direction. For waves radiated to the $-\theta$, a similar discussion holds.

Thus, the factor contributing to the radiation pattern is simply written as

$$
w_{R}^{T}(\varphi, b)=j \cdot C_{1} \cdot b G_{n}(b)-C_{2} \cdot n j_{n}(k b) .
$$

Equation (28) is valid for any radial modes of order $n$, as long as the restriction $n \gg 1$ is satisfied.

Some familiar formulas in mathematical physics (e.g., WATSON, 1966) are introduced in order to reduce Eq. (28) to a more convenient form to compare the radiation pattern of the toroidal modes with that of $S H$ waves given by Eq. (5).

$$
J_{\nu}(\nu \sec \alpha)=\frac{\cos [\nu(\tan \alpha-\alpha)-\pi / 4]}{\sqrt{(1 / 2) \cdot \nu \pi \cdot \tan \alpha}}, \quad(0<\alpha<\pi / 2) .
$$

Modifying this formula, we have

$$
J_{\nu-1}(\nu \sec \alpha)=\frac{\cos [\nu(\tan \alpha-\alpha)-\pi / 4+\alpha]}{\sqrt{(\pi / 2) \cdot(\nu \tan \alpha+\cot \alpha)}},
$$

Equations (29) and (30) are the asymptotic formulas valid for large values of $\nu$.

Putting

$$
\left.\begin{array}{l}
\nu=n+1 / 2, \\
\nu \cdot \sec \alpha=\xi,
\end{array}\right\}
$$

and assuming

$$
\xi \gg \nu,
$$

we have

$$
\begin{gathered}
j_{n}(\xi) / \xi=\frac{1}{\xi^{3 / 2} \cdot\left(\xi^{2}-\nu^{2}\right)^{1 / 4}} \cdot \cos \chi, \\
\frac{d}{d \xi}\left(j_{n}(\xi) / \xi\right)=-\frac{1}{\xi^{8 / 2} \cdot\left(\xi^{2}-\nu^{2}\right)^{1 / 4}} \cdot \frac{\sqrt{\xi^{2}-\nu^{2}}}{\xi} \cdot \sin \chi,
\end{gathered}
$$

where 


$$
\chi=\sqrt{\xi^{2}-\nu^{2}}-\nu \cdot \cos ^{-1}(\nu / \xi)-\pi / 4 .
$$

In deriving Eqs. (33) and (34), the well-known relation between $j_{n}(\xi)$ and the Bessel function $J_{\nu}(\xi)$, and the recurrence formuta are employed. Putting

$$
\xi=k b,
$$

and employing Eqs. (25), (33) and (34) under the conditions in Eq. (32), Eq. (28) is reduced to

$$
\begin{aligned}
w_{R}^{T}(\varphi, b)= & \left\{1 / 2 \xi^{1 / 2}\left(\xi^{2}-\nu^{2}\right)^{1 / 4}\right\} \cdot\left[\left\{-C_{1} \cdot \sqrt{\xi^{2}-\nu^{2}}-C_{2} \cdot n\right\} \cdot e^{j x}\right. \\
& \left.+\left\{C_{1} \cdot \sqrt{\xi^{2}-\nu^{2}}-C_{2} \cdot n\right\} \cdot e^{-j x}\right] .
\end{aligned}
$$

It is immediately found that Eq. (37) is similar in form to Eq. (13) which gives the Love wave displacement. This leads us to suppose that the each term in the brackets may have some connection with a particular ray as was seen in the previous case for Love waves. To prove this, the method developed in the preceding section is applied, comparing the radiation pattern of respective waves in the brackets with that of $S H$ waves in Eq. (5). Defining $i_{h}^{T}$ by

$$
\tan i_{h}^{T}=\frac{n}{\sqrt{\xi^{2}-\nu^{2}}}
$$

and using Eq. (5), Eq. (37) can be transformed to

$$
w_{R}{ }^{T}(\varphi, b)=\left\{\left(\xi^{2}-\nu^{2}\right)^{1 / 4} / 2 \xi^{1 / 2} \cdot \cos i_{h}^{T}\right\} \cdot\left[R_{S H}\left(i_{h}{ }^{T}, \varphi\right) \cdot e^{j x}+R_{S H}\left(\pi-i_{h}^{T}, \varphi\right) \cdot e^{-j x}\right] .
$$

The angle $i_{h}{ }^{T}$, the value of which is limited within the range $(0, \pi / 2)$, is called the equivalent take-off angle for the torsional oscillation, because the radiation pattern of respective waves composing the toroidal displacement is just the one of $S H$ waves associated with this angle as is apparent from Eq. (39).

The numerator of Eq. (38) may be replaced by $\nu$ for large $n$ with sufficient accuracy. With this and Eqs. (31) and (36), the following important formulas are obtained

$$
\sin i_{h}{ }^{T}=\nu / \xi=(n+1 / 2) / k b=(n+1 / 2) \cdot v s / p \cdot b .
$$

Then the ray parameter becomes

$$
\frac{b \cdot \sin i_{h}^{T}}{v_{S}}=\frac{a \cdot \sin e_{0}}{v_{S}}=\frac{r_{d}}{v_{S}}=\frac{n+1 / 2}{p}=\frac{a}{C_{T}}
$$

where $e_{0}$ denotes the angle of incidence at the free surface, $r_{d}$ the radius of the deepest point of the ray ( $c f$. Fig. 5). $C_{T}$ is the phase velocity for the torsional oscillation defined by

$$
C_{T}=\frac{k a}{n+1 / 2} \cdot v \mathrm{~S}
$$

It must be noted that the quantities $p, i_{h}{ }^{T}, e_{0}, r_{d}$, and $C_{T}$ are the functions of radial mode and colatitudinal order numbers. 


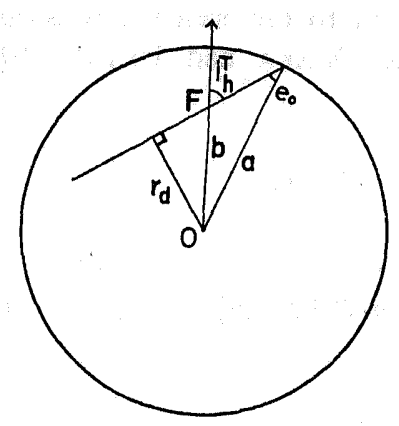

Fig. 5. The ray geometry in the homogeneous sphere. $O$ is the center of the sphere and $F$ the focus. The radius of the earth is denoted by $a$ and the radius to the source surface is $b$.

Equation (41) has been derived by BeN-MeNAHEM (1964) applying the analytical method to the normal mode solution. This relation connects particular modes with particular rays through the identity of the phase velocity of normal modes and the apparent velocity of $S H$ waves. BRUNE (1964) calculated the phase velocity of toroidal modes from the body wave phases assuming this relation.

It is significant that Eq. (40) or (41) relating modes and rays is now established by a quite different method based on the idea that both radiation patterns should have some connection each other.

Thus, it is found that the normal mode solution for the torsional vibration with any mode and order numbers can be expressed by the summation of two terms associated with the particular set of rays, the situation of which is quite similar to the Love wave case. However, some conditions have to be satisfied for the above discussion to be valid. These are written

$$
k b \gg n+1 / 2 \gg 1 \text {. }
$$

Referring to the numerical results for the eigen-frequencies of the torsional oscillation of several earth models presented by SATô and his co-workers (SATÓ and USAMr, 1962; SATô et al., 1963; SATô et al., 1968), it can be seen that Eq. (43) is generally satisfied in higher torsional modes with a large order number $n$ as long as the focal depth is not large. This is not inconsistent with the restriction assigned by BENMenahem (1964).

Here, it must be said that it is impossible to specify the particular set of rays by comparing the observed radiation pattern of normal modes with the theoretical body wave pattern, because the actual radiation pattern is not either one of the two terms in Eq. (39) but the resultant pattern of the two. In the next section, the radiation pattern for the torsional oscillation of a radially heterogeneous sphere will be generally treated.

\section{Radiation Pattern of Toroidal Modes}

So far, the azimuthal dependence of displacement of normal mode solutions and the mode-ray relation have been treated for simple cases. In this section, the radiation pattern of the torsional displacement of a radially heterogeneous sphere excited by a double-couple force system is discussed, and the applicability of the mode-ray relation obtained for the homogeneous sphere is considered. 
The expression of the azimuthal displacement relevant to the present case is easily derived from formulas given by Usami et al. (1970) and ODAKA and UsAmI (1970). This is written in the form

$$
\begin{aligned}
w_{H}(r, \theta, \varphi, t)= & -\frac{j \cdot M_{0}}{2 \pi \mu_{b} a^{2} b} \sum_{i, n} \frac{2 n+1}{n(n+1)}\left\{\frac{f^{*}(p) \cdot e^{j p t}}{\frac{\partial G_{w}(a)}{\partial p}} \cdot W_{n}(r)\right. \\
& \left.\cdot\left[C_{1} \cdot b G_{w}(b) \cdot \dot{P}_{n}{ }^{1}(\cos \theta)+C_{2} \cdot W_{n}(b) \cdot \dot{P}_{n}{ }^{2}(\cos \theta)\right]\right\}_{p=i_{i} p_{n}},
\end{aligned}
$$

where

$$
G_{w}(r)=\frac{d W_{n}(r)}{d r}-\frac{W_{n}(r)}{r}=r \frac{d}{d r}\left(\frac{W_{n}(r)}{r}\right)
$$

$W_{n}(r)$ denotes the eigen-function giving the radial distribution of the azimuthal displacement, the value of which is normalized to unity at the free surface, and $G_{w}(r)$ the quantity giving the azimuthal stress. The characteristic equation for the torsional vibration of the radially heterogeneous sphere is $G_{w}(a)=0 . \mu_{b}$ is the rigidity at the source depth and a dot $(\cdot)$ over the associated Legendre function stands for $d / d \theta$. Other quantities such as $M_{0}, a, b, C_{1}, C_{2}$ and $f^{*}(p)$ are defined in previous sections.

Comparing the above two expressions with those for the homogeneous sphere given by Eqs. (24) and (25), it is immediately noticeable that the latter are entirely included in the former. On the other hand, a radially heterogeneous sphere can be considered to consist of thin spherical homogeneous shells. Therefore, $W_{n}(r)$ is supposed to be approximated in respective shells by the solution valid for the homogeneous medium.

These aspects suggest that a discussion similar to the homogeneous sphere holds for the present case as well. So it can be concluded that Eqs. (40) and (41) are also valid for the radially heterogeneous sphere. The shear wave velocity $v_{S}$ appearing in them, then, varies as a function of the radius.

It is instructive to examine the radial distribution of the azimuthal displacement of normal modes and the deepest point of the corresponding ray through Eq. (41), because if such a mode-ray relation is true, it is reasonable to consider that the deepest point should correspond to the lowermost part of the displacement distribution. Figure 6 illustrates these situations in which the function $W_{n}(r)$ for higher radial modes with a large order number is displayed for a Gutenberg-Bullen $\mathrm{A}^{\prime}$ earth model. The deepest points associated with respective normal modes calculated by Eq. (41) are shown by arrows. It is certain from the figure that the points indicated by these arrows are located just below the lowermost maximum amplitude. In other words, the energy associated with respective normal modes hardly penetrates into the medium inner than the envelope of the ray given by Eq. (41). These features can be recognized not only for higher modes and large order numbers as indicated here but also for lower modes and small orders such as $i=2$ with $n=20$ and also for a model with a homogeneous mantle and a liquid core as well. Therefore, the validity of Eqs. (40) and (41) may be extended to the first overtone and low order numbers. The data used here are those prepared by Satô and Usami for the theoretical study of the wave generation, propagation, and attenuation.

A convenient formula to investigate the radiation pattern of the torsional displace- 

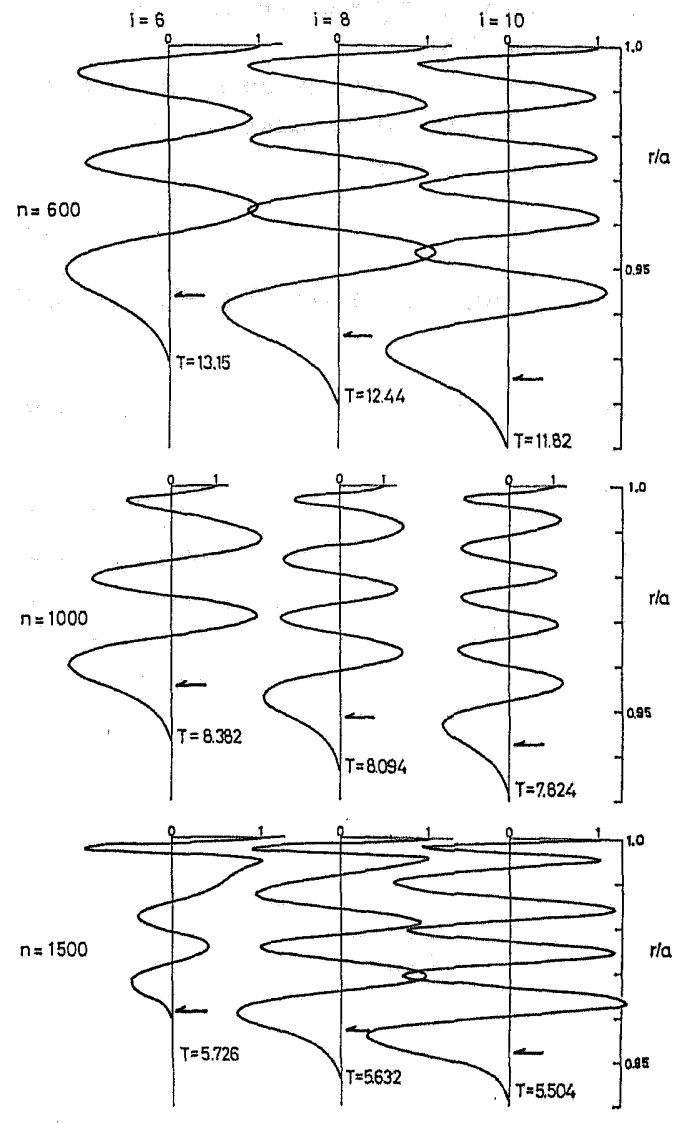

Fig. 6. Radial distribution of azimuthal displacements for radial higher toroidal modes with large colatitudinal order numbers, which are normalized at the surface. The Gutenberg-Bullen $\mathrm{A}^{\prime}$ spherical earth is assumed. The radial mode number is $i . n$ is the colatitudinal order number and $T$ the eigen-period. Arrows show the deepest point of the ray corresponding to each normal mode by means of Eq. (41).

ment spectra can be obtained by transforming the quantity in the brackets in Eq. (44) using the asymptotic expansion of the associated Legendre function for a large colatitudinal order number $n$.

$$
\begin{aligned}
C_{1} \cdot b G_{w}(b) \cdot \dot{P}_{n}^{1}(\cos \theta)+C_{2} \cdot W_{n}(b) \cdot \dot{P}_{n}^{2}(\cos \theta), \\
\quad=C_{1} \cdot b G_{w}(b) \cdot n^{2} \cdot k_{n} \cdot \sin \Theta-C_{2} \cdot W_{n}(b) \cdot n^{3} \cdot k_{n} \cdot \cos \Theta, \\
\left.=\left(n^{2} \cdot k_{n}(2) \cdot R_{T^{w}}(b, \varphi) \cdot\left[e^{j\left(\Theta+\beta_{n}(w)\right.}-\pi\right)+e^{-j\left(\Theta+\beta_{n}(w)+\pi\right)}\right)\right],
\end{aligned}
$$

where

$$
R_{T} w(b, \varphi)=\left[\left\{C_{1} \cdot b G_{w}(b)\right\}^{2}+\left\{C_{2} \cdot W_{n}(b) \cdot n\right\}^{2}\right]^{1 / 2},
$$

and

$$
\tan \beta_{n}{ }^{(w)}=b G_{w}(b) \cdot C_{1} / n W_{n}(b) \cdot C_{2} .
$$

$k_{n}$ and $\Theta$ are the same as in Eq. (27).

The function $R_{T^{w}}(b, \varphi)$ determines the azimuthal variation of amplitude spectrum at frequency ${ }_{i} p_{n}$ due to a double-couple point source in the radially heterogeneous 
sphere. For a homogeneous one, it is necessary only to replace the function $W_{n}(b)$ by $j_{n}(k b)$ and $G_{v v}(b)$ by $G_{n}(b)$ in Eqs. (46), (47), and (48).

Comparing Eqs. (47) and (6), it is readily found that there is an apparent equivalent take-off angle $\bar{\tau}_{h}^{T}$ which makes identical the azimuthal variation of both quantities denoted by $E_{S H}\left(\bar{l}_{h}{ }^{T}, \varphi\right)$ and $\left(R_{T^{2 v}}(b, \varphi)\right)^{2}$. This is defined by

$$
\tan ^{2} \bar{\tau}_{h}^{T}=\left\{n W_{n}(b) / b G_{w}(b)\right\}^{2},
$$

where the value of $\bar{\imath}_{h}{ }^{T}$ is limited within the range $(0, \pi / 2)$. From Eqs. (6), (47), and (49), the following relation is derived

$$
\left\{R_{T}^{w}(b, \varphi)\right\}^{2}=\left\{b G_{w}(b) / \cos \bar{l}_{h}^{T}\right\}^{2} \cdot E_{S H}\left(\bar{l}_{h}^{T}, \varphi\right),
$$

similar to Eq. (21) for the Love wave case.

The above relation indicates that the radiation pattern of square amplitude for toroidal modes is given by the quantity associated with the $S H$ wave energy emitted in particular directions specified by the take-off angle $\bar{l}_{h}^{T}$. This angle generally differs from the equivalent take-off angle $i_{h}{ }^{T}$ introduced in the previous section and can be defined even for the fundamental radial modes, but does not seem to have any physical significance except for the coincidence of radiation patterns. This, however, has some

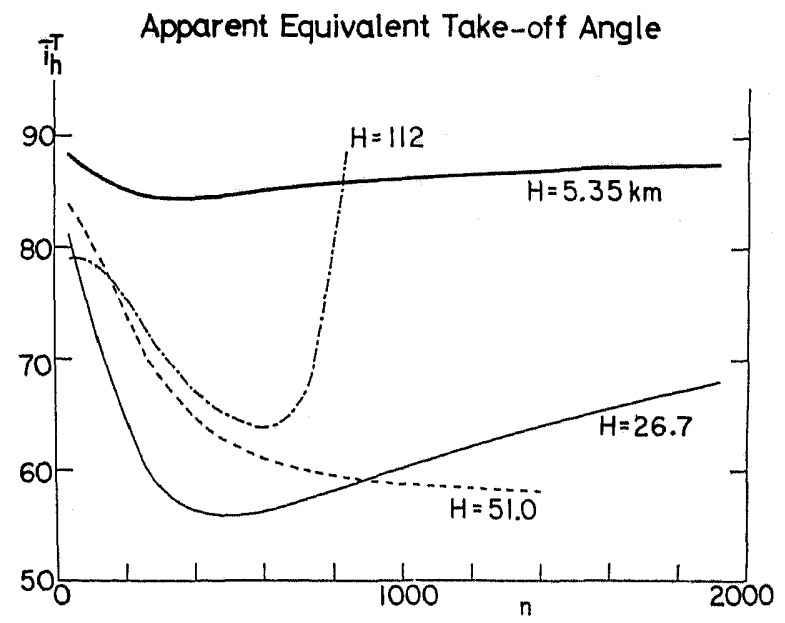

Fig. 7. Numerical examples of the apparent equivalent take-off angle $\bar{\tau}_{h}{ }^{T}$ calculated for the torsional oscillation of the Gutenberg-Bullen $\mathrm{A}^{\prime}$ earth model. Only fundamental radial mode $i=1$ is taken into account, which is considered to contribute mainly to surface waves. The ordinate indicates the angle in degree and the abscissa the colatitudinal order number. $H$ means the focal depth.

advantages in calculating the radiation pattern of amplitude spectra of normal modes, because the pattern can be evaluated from the function $E_{S H}\left(i_{h}, \varphi\right)$ given by Eq. (6), provided that the apparent equivalent take-off angles associated with those modes are known beforehand.

Some numerical examples will be demonstrated here for the fundamental radial modes by using the data for the Gutenberg-Bullen $\mathrm{A}^{\prime}$ spherical earth computed by SATô 


\section{RADIATION PATTERN OF TORSIONAL MODE $i=1$ source depth $=5.35 \mathrm{~km}$}
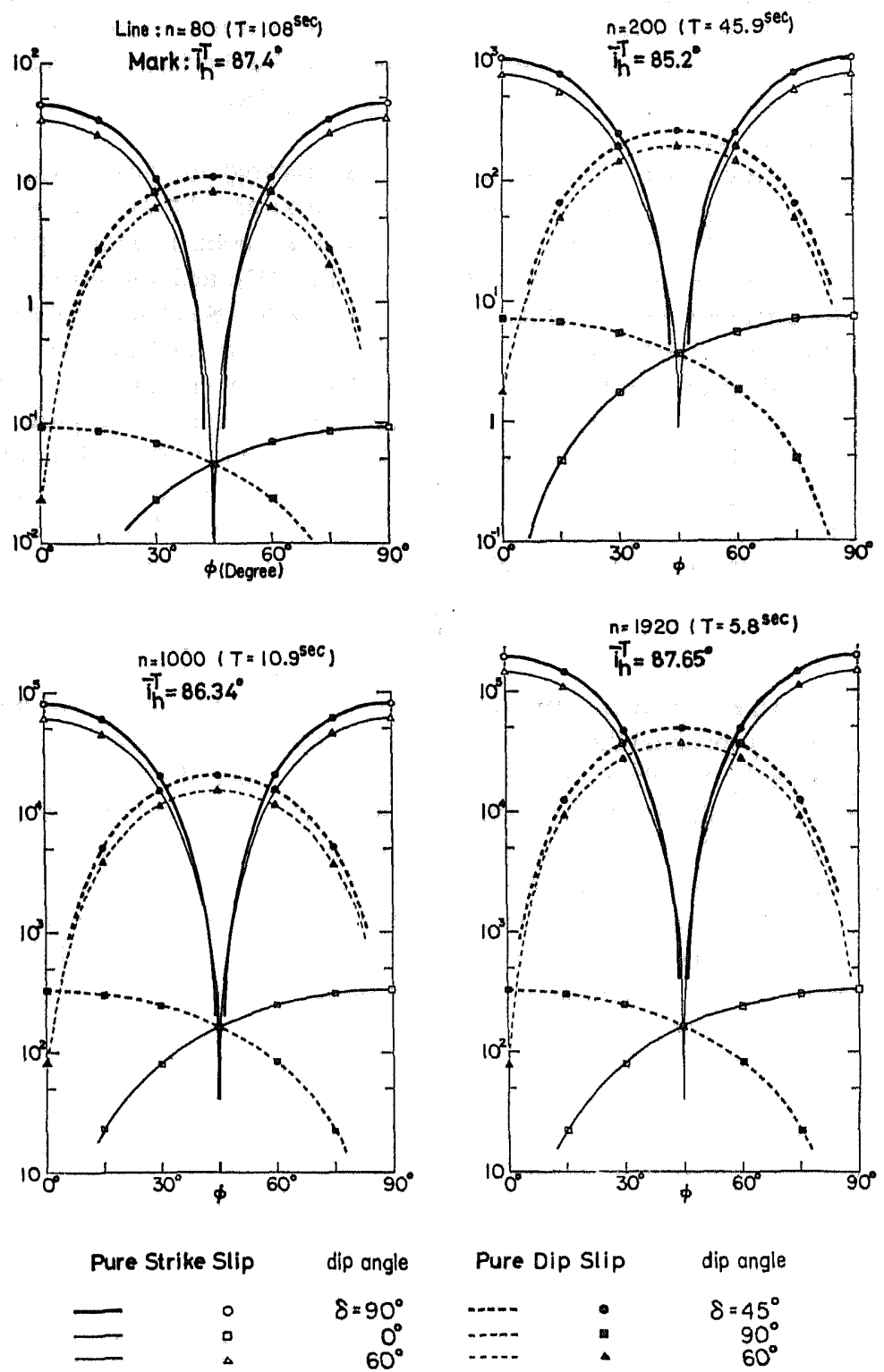

Fig. 8. Radiation pattern of azimuthal displacements for several fundamental toroidal modes excited by pure dip slip and strike slip faults at depth $5.35 \mathrm{~km}$ in the Gutenberg-Bullen $\mathrm{A}^{\prime}$ earth model.

et al. (1968). In Fig. 7 is shown the apparent equivalent take-off angle calculated for several focal depths by Eq. (49). It is seen from the figure that the radiation pattern 
varies remarkably as a function of the colatitudinal order number. The angle, however, seems to approach some constant value independent of order numbers as the focal depth becomes shallower. It can be readily found from Eq. (49) that the extreme value of this angle is $\pi / 2$, remembering that $G_{w}(r)$ is zero and $W_{n}(r)$ unity at the free surface. In other words, the apparent ray tends to the horizontal direction as the focus closes to the surface. A similar feature is recognized for the Love wave case discussed in Section 2.

Once the apparent equivalent take-off angle is prepared for any modes and focal depths, the radiation pattern of normal modes for arbitrary dip and slip is obtained by calculating that of $S H$ waves. Figure 8 manifests that both radiation patterns, one calculated by a conventional method based on Eq. (47) and the other by Eq. (50) employing the apparent angle of Fig. 7, agree very well. Solid and broken lines show those by the former method. Symbols such as circles, squares, and triangles are obtained by the latter method at every $15^{\circ}$ of the azimuth. Broken lines and solid marks denote the pure dip slip case and solid lines and open marks the pure strike slip. Calculation is carried out for the azimuth within the range $\left(0^{\circ}, 90^{\circ}\right)$. From this figure, the radiation pattern for the azimuth $\left(90^{\circ}, 360^{\circ}\right)$ is obtained using the symmetrical relations of the amplitude spectra (ODAKA and USAMI, 1970). Thus, it will be useful to prepare lists of the apparent equivalent take-off angle for various earth models.

\section{Conclusion}

Eq. (41) obtained by Ben-MeNaHem (1964) was also derived by comparing the radiation pattern of torsional displacement spectra with that of $S H$ waves. This relation was also supported by the close correlation between the radial distribution of azimuthal displacements of toroidal modes and the deepest points of the corresponding rays assigned by this formula. Considering these results together with the fact that BRUNE (1964) has successfully obtained the torsional phase velocity from the body wave phases, it may be concluded that this relation is just the one that connects modes and rays as far as the torsional modes are concerned. It then seems that this is applicable for any higher modes of order numbers larger than 20 or so.

These features, however, cannot be said to be completed until theoretical seismograms synthesized taking this relation into consideration reveal the various $S H$ wave phases.

The author would like to express his sincere thanks to Professor Tatsuo Usami for kindly reading the munuscript and for the use of his numerical data.

\section{REFERENCES}

Alterman, Z., and P. Kornfeld, Normal modes and rays in the propagation of a seismic pulse from a point-source in a layered sphere, Israel J. Tech., 4, 198-213, 1966.

Alterman, Z., and J. ABoudi, Seismic pulse in a layered sphere: Normal modes and surface waves, J. Geophys. Res., 74, 2618-2636, 1969.

Ben-Menahem, A., Mode-ray duality, Bull. Seismol. Soc. Amer., 54, 1315-1321, 1964.

Ben-Menahen, A., S.W. Smith and T.L. Teng, A procedure for source studies from spectrums of long-period seismic body waves, Bull. Seismol. Soc. Amer., 55, 203-235, 1965.

Brune, J. N., Travel times, body waves, and normal modes of the earth, Bull. Seismol. Soc. 
Amer., 54, 2099-2128, 1964.

EwING, W.M., W.S. JARDetzky and F. PRess, "Elastic Waves in Layered Media," McGrawHill, New York, 1957.

HASKell, N. A., Radiation pattern of surface waves from point sources in a multi-layered medium, Bull. Seismol. Soc. Amer., 54, 377-393, 1964.

Landisman, M., T. Usami, Y. Satô and R. MAssé, Contributions of theoretical seismograms to the study of modes, rays, and the earth, Rev. Geophys. Space Phys., 8, 533-589, 1970.

ODAKA, T., and T. USAMI, Theoretical seismograms and earthquake mechanism, III. Azimuthal variation of the initial phase of surface waves, Bull. Earthq. Res. Inst., 48, 669689, 1970.

Officer, C. B., "Introduction to the Theory of Sound Transmission; with Application to the Ocean," McGraw-Hill, New York, 1958.

SATo, R., Formulations of solutions for earthquake source models and some related problems, J. Phys. Earth, 17, 101-110, 1969.

SArô, Y., Study on surface waves I. Velocity of Love-waves, Bull. Earthq. Res. Inst., 29, 1-11, 1951.

SATô, Y., Study on surface waves X. Equivalency of $S H$-waves and sound waves in a liquid, Bull. Earthq. Res. Inst., 32, 7-16, 1954.

SATô, Y., and T. USAMI, Basic study on the oscillation of a homogeneous elastic sphere (I-III), Geophys. Mag., 31, 15-62, 1962.

SATO, Y., T. USAMr, M. LANDISMAN and M. EwING, Basic study on the oscillation of a sphere (V), Propagation of torsional disturbances on a radially heterogeneous sphere, Case of a homogeneous mantle with a liquid core, Geophys. J.R.A.S., 8, 44-63, 1963.

SATÓ, Y., T. USAMI and M. LANDISMAN, Theoretical seismograms of torsional disturbances excited at a focus within a heterogeneous spherical earth-Case of a Gutenberg-Bullen $\mathbf{A}^{\prime}$ earth model, Bull. Seismol. Soc. Amer., 58, 133-170, 1968.

USAMI, T., T. ODAKA and Y. SATÔ, Theoretical seismograms and earthquake mechanism, I. Basic principle, II. Effect of time function on surface waves, Bull. Earthq, Res. Inst., 48, 533-579, 1970.

Watson, G. N., "A Treatise on the Theory of Bessel Function," 2nd Ed., Cambridge Univ. Press, 1966. 